\title{
Exploring spatial dependencies in the prevalence of childhood diarrhea in Mozambique using global and local measures of spatial autocorrelation
}

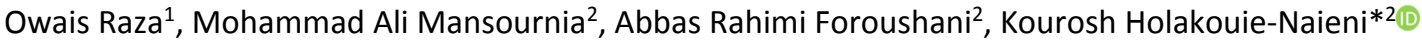 \\ Received: 1 Oct 2018 \\ Published: 8 Jun 2020
}

\section{Abstract}

Background: Diarrhea in children under 5 years is generally considered as an important public health problem and the major cause of childhood morbidity and mortality in Sub-Saharan Africa. The purpose of this study was to present exploratory spatial data analyses to identify spatial clusters and outliers in the prevalence of childhood diarrhea in Mozambique.

Methods: Using data from 2011 Mozambique Demographic and Health Survey, we calculated the prevalence of childhood diarrhea on the district level. Two exploratory spatial data analyses methods were applied, namely, global and local Moran's I statistics, providing spatial autocorrelation and spatial clusters/outlier in the prevalence of childhood diarrhea, respectively.

Results: Choropleth mapping and global Moran's I statistics showed that the prevalence of childhood diarrhea has clustered distribution across the study area. A local Moran's I index revealed spatial clusters within the province of Tete, Gaza, Cabo Delgado, and Zambezia. However, spatial outliers were confined within Sofala province.

Conclusion: The exploratory spatial data analyses showed various spatial clustering and outliers present in the prevalence of childhood diarrhea, indicating interventions needed in targeted regions.

Keywords: Diarrhea, Mozambique, Geographic information systems, Spatial analysis

Conflicts of Interest: None declared

Funding: Tehran University of Medical Sciences

\section{*This work has been published under CC BY-NC-SA 1.0 license. \\ Copyright $₫$ Iran University of Medical Sciences}

Cite this article as: Raza O, Mansournia MA, Rahimi Foroushani A, Holakouie-Naieni K. Exploring spatial dependencies in the prevalence of childhood diarrhea in Mozambique using global and local measures of spatial autocorrelation. Med J Islam Repub Iran. 2020 (8 Jun);34:59. https://doi.org/10.47176/mjiri.34.59

\section{Introduction}

The public health significance and impact of childhood diarrhea is substantial, resulting in 525000 deaths each year (1). This puts diarrhea among 5 leading cause of childhood morbidity and mortality and an urgent health priority. In developing countries, diarrheal disease is responsible for $18 \%$ to $20 \%$ of all deaths in children younger than 5 years. Among World Health Organization

Corresponding author: Dr Kourosh Holakouie-Naieni, holakoin@tums.ac.ir

\footnotetext{
1. School of Public Health, Dow University of Health Sciences, Karachi, Pakistan 2. Department of Epidemiology and Biostatistics, School of Public Health, Tehran University of Medical Sciences, Tehran, Iran
}

(WHO) regions, the burden of childhood diarrhea is especially highest in South Asia and Sub-Sahara Africa (2-4). In pursue of Millennium Development Goals (MDGs), childhood mortality attributed to diarrheal diseases has been declined since 2000 .

Status of child health is a crucial indicator of the quality of life (5); therefore, examining the prevalence and pat-

$\uparrow$ What is "already known" in this topic:

Autocorrelation simply means that a variable is correlated with itself, ie, the pairs of observations that are close to each other are more likely to have values that are more similar, and pairs of observations far apart from each other are more likely to have values that are less similar. It is known in literature that many health outcomes possess spatial autocorrelation.

\section{$\rightarrow$ What this article adds:}

We applied methods of global and local spatial autocorrelation to identify the areas where high and low prevalence of childhood diarrhea are concentrated in Mozambique and to identify regions that need more attention in terms of resource allocation. 
terns of childhood diarrhea at subnational level are rewarding efforts in identifying high-risk areas where further interventions could be directed. In Mozambique, diarrhea continues to be an endemic childhood disease, with seasonal (6) and geographical variations (7). According to the Demographic and Health Survey (DHS-2011), 11.1\% of children younger than 5 years had at least one diarrheal episode within the past 2 weeks of survey (8). This survey also reported that the prevalence of diarrhea considerably varies at subnational level, indicating spatial heterogeneity in the prevalence of disease.

Like any other infectious diseases, in childhood diarrhea, host, agent, human and physical environment are codependent and manifest spatial relationship (9). Hence, it is of utmost importance to study geographical variation of prevalence of childhood diarrhea and spatial dependency (i.e. the lack of independence in data at locations which are close together) at local level. A spatial analytical method can provide a measure that can suggest spatial dependence or spatial independence (10). By recognizing the areas with spatial dependency, vulnerable areas for future outbreaks of diarrheal diseases can be identified. It is necessary to identify spatial patterns in form of clusters and outliers. A spatial cluster is an area considered by feature (i.e. variable of interest) with either high or low values surrounded by neighboring areas demonstrating similar high or low values for the same feature. However, spatial outlier is an area where a feature demonstrate statistically significant higher or lower values as compared to the values found in neighboring areas (11). Spatial differences in childhood morbidity have been reported from many countries in Sub-Saharan Africa (12-16). Using various spatial analytical methods, studies have determined spatial heterogeneity in the prevalence of childhood morbidity and its associated biodemographic and socioeconomic risk factors. Therefore, the objective of this study was to apply spatial analytical techniques to identify spatial autocorrelation, spatial cluster, and spatial outliers in the prevalence of childhood diarrhea in Mozambique.

\section{Methods \\ Data}

This study analyzed the data from Mozambique Demographic and Health Survey (DHS) - 2011. Mozambique is a southeastern African country bordered with Tanzania, Malawi, Zimbabwe, South Africa, and Indian Ocean on Eastern border (Fig. 1). The Mozambique DHS 2011 was the third and the latest survey (at the time of data acquisition by the authors) conducted as a part of the MEASURE DHS program international series. In this survey, a 2staged sampling method was implemented in a way that the final sample was representative at national level, subnational level, rural, and urban areas. All urban and rural areas were divided into smaller areas known as Enumeration Area (EA). Within each EA, list of households served as the sampling frame for the selection of household for the second stage. In the second stage of sampling, fixed number of households was selected through systematic sampling technique. A detailed note on DHS sampling technique can be found in the Appendix $\mathrm{A}$ of
Moçambique Inquérito Demográfico e de Saúde 2011 (8). The survey collected detailed information relating to demographic, child health care, and GPS coordinates from each EA. With a response rate of $99 \%, 13,745$ evermarried women age 15-49 years were interviewed. We extracted the information regarding the episode of diarrhea from every mother with a child aged 0-59 months. The data were then exported to Esri ArcGIS 10.1 software, where data table was first merged with the DHS clusters and then aggregated for each subnational level.

\section{Global spatial autocorrelation}

The global measure of spatial autocorrelation was developed by Moran P (17), which measures the spatial dependence of the variable. The spatial dependence denotes lack of independence in data in relation to its neighboring data points (18). Therefore, measure of spatial autocorrelation may suggest spatial dependence (clustering) or spatial independence (dispersed). To test spatial dependence, the Moran's I index formula is given as follows:

$$
I=\frac{n}{\sum_{i=1}^{n} \sum_{j=1}^{n} w_{i j}} \cdot \frac{\sum_{i=1}^{n} \sum_{j=1}^{n} w_{i j}\left(x_{i}-\bar{x}\right)\left(x_{j}-\bar{x}\right)}{\sum_{j}\left(x_{i}-\bar{x}\right)}
$$

where $\mathrm{n}$ is the number of data points $x_{i}$ is the proportion of children with diarrhea at location $i^{\text {th }}$ (where $i^{\text {th }}$ location denotes location of interest), $x_{j}$ is the proportion of children with diarrhea at location $\mathrm{j}$ (where $\mathrm{j}^{\text {th }}$ location refers to neighboring locations around $\mathrm{i}^{\text {th }}$ location), $\bar{x}$ is the mean proportion of children with diarrhea and $w_{i j}$ is the spatial weights applied between location $i$ and $j$. These spatial weights are assigned in a manner that $j^{\text {th }}$ neighbours receive a weight of 1 if their boundaries are adjacent to the $\mathrm{i}^{\text {th }}$ location, otherwise not. Similar to correlation coefficient, the value of Moran's I ranges between -1.0 and +1.0 . To test the null hypothesis that there is no spatial dependence across the study area, the following equation was used:

$$
Z=\frac{I-E(I)}{\sqrt{\operatorname{var}(I)}}
$$

where $\mathrm{E}(\mathrm{I})$ represents the expected value of observed Moran's I, var(I) represents the variance of Moran's I. Given a significant $\mathrm{p}$ value, a positive Moran's I denotes that the spatial distribution of high values and/or low values in the dataset are more spatially clustered than would be expected if underlying spatial processes were random. However, a significant negative Moran's I index means that the spatial distribution of high values and low values in the dataset are more spatially dispersed than would be expected if underlying spatial processes were random. The tool for calculating spatial autocorrelation in Esri ArcGIS 10.1 returns Moran's I index and its associated $\mathrm{p}$ values. 


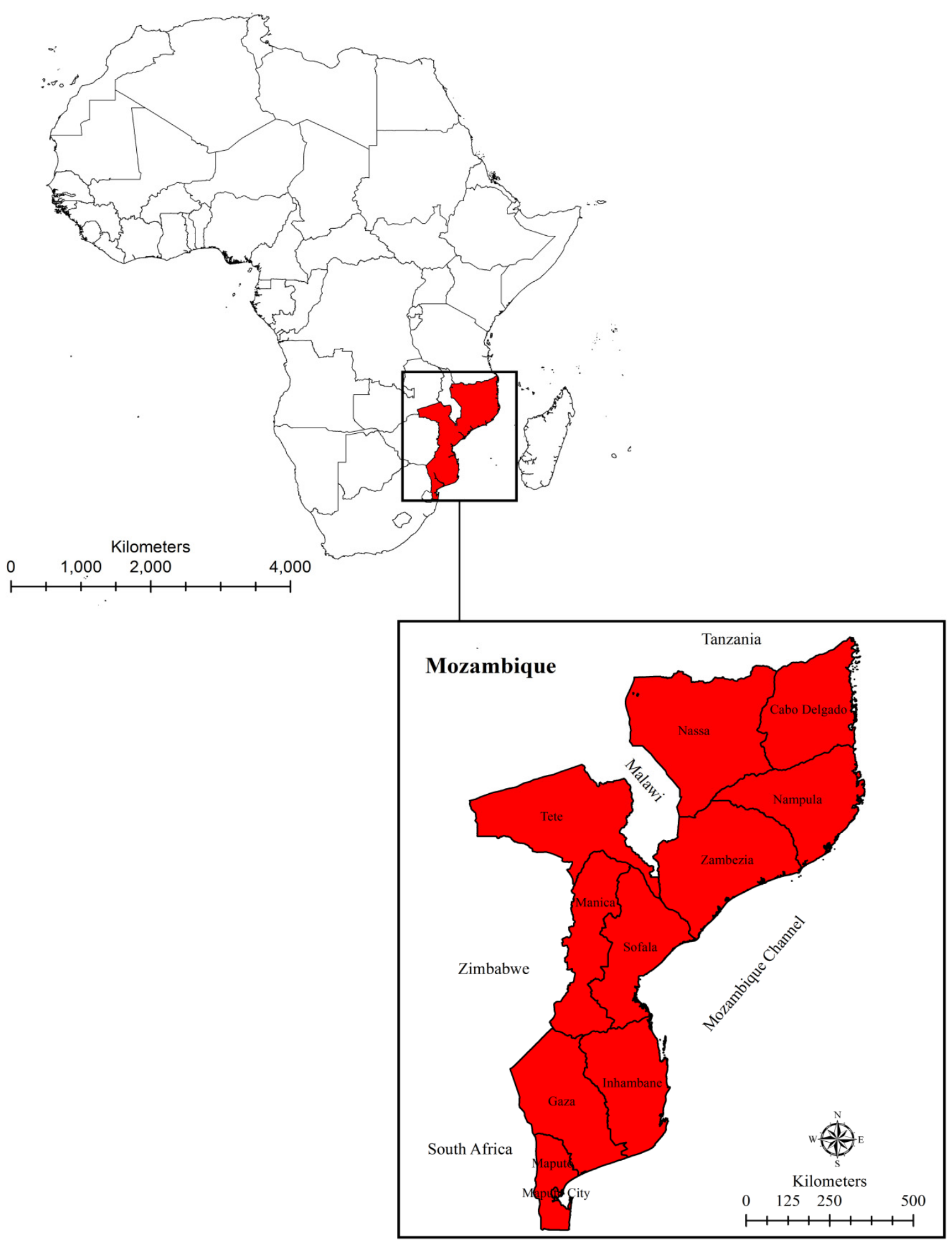

Fig. 1. Location of study area

\section{Local spatial autocorrelation}

To identify spatial clusters and outliers in the study area, Anselin local Moran's I (also called LISA - a Local Indicator of Spatial Autocorrelation) was applied. In essence, local Moran's I allows decomposition of global Moran's I statistics over the study region to assess spatial concentra-

tions in population subgroups (19). In addition to identifying spatial cluster, this index also assesses the influence of individual locations on the magnitude of the global Moran's I index. The local Moran's I index for the $\mathrm{i}^{\text {th }}$ region is calculated by the following formula: 


$$
I_{i}=\frac{x_{i}-\bar{x}}{S_{i}^{2}} \sum_{j=1, j \neq i}^{n} w_{i, j}\left(x_{j}-\bar{x}\right)
$$

where $x_{i}$ is the proportion of children with diarrhea at location $\mathrm{i}, \bar{x}$ is the mean proportion of children with diarrhea, $w_{i j}$ is the spatial weights applied between location i and $\mathrm{j}$ (as explained above), and $S_{i}^{2}$ is an estimate of the variance and is equal to:

$$
S_{i}^{2}=\frac{\sum_{j=1, j \neq i}^{n}\left(x_{j}-\bar{x}\right)^{2}}{n-1}-\bar{x}^{2}
$$

A positive value of local Moran's index I denotes that a variable is surrounded by similar high or low values and forms a spatial cluster, while a negative value indicates that a variable has neighbours with dissimilar values (20). The local Moran's I was tested using the following Zscore test:

$$
Z=\frac{I_{i}-E\left(I_{i}\right)}{\sqrt{\operatorname{var}\left(I_{i}\right)}}
$$

where $I_{i}$ is the observed local Moran's I index for $\mathrm{i}^{\text {th }}$ location and $E\left(I_{i}\right)$ is the expected value of $\mathrm{I}$ for the $\mathrm{i}^{\text {th }}$ location. The null hypothesis for local Moran's I states that there is no association between prevalence of childhood diarrhea of a region with its neighboring regions, whereas the alternate hypothesis supports that there is a similarity (spatial cluster) or dissimilarity (spatial outlier) in the prevalence of childhood diarrhea (20). The tool for calculating local Moran's I index in Esri ArcGIS 10.1 returns local Moran's I index with associated Z scores and $\mathrm{p}$ values for each location. In ArcGIS environment, $\mathrm{Z}$ scores and associated $p$ values are calculated by the Randomization Null Hypothesis testing. This hypothesis test postulates that the observed spatial pattern of the data represents one of many (n!) possible spatial arrangements. According to the $\mathrm{z}$-score and its associated $\mathrm{p}$ value, regions are divided into 5 spatial association types: (1) a region with high prevalence of childhood diarrhea surrounded by regions with high prevalence (High-High cluster) with zscore greater than 1.96; (2) a region with low prevalence which is bounded by neighboring regions with low prevalence as compared to the average (Low-Low cluster) with $z$-score greater than 1.96; (3) a region where prevalence is higher than the average and is surrounded by region of low prevalence (High-Low outlier) with z-score less than 1.96 ; (4) a region with prevalence is less than the average while neighboring regions have a prevalence greater than the average (High-Low outlier) with z-score less than 1.96; and (5) a region where prevalence of childhood diarrhea is not significantly different from the average. Among these, high-high and low-low types make spatial clusters pattern of prevalence, while high-low and lowhigh types make spatial outliers. These high and low types are in relation to the average value of the prevalence of childhood diarrhea in the study region.

\section{Results}

The overall prevalence of childhood diarrhea in Mozambique is $11.1 \%$. The choropleth map of prevalence of childhood diarrhea in Mozambique, stratified by regions and classified into 5 categories, is presented in Figure 2 (a). This choropleth map demonstrates the highest and lowest prevalence of childhood diarrhea across study area. Higher prevalence of childhood diarrhea was found in the regions within Tete, Zambezia, Sofala, and Nassa. On the other hand, regions bordering Mozambique Channel in the southeast of Mozambique mainly demonstrated lower prevalence.

Moran's I index of global spatial autocorrelation for childhood diarrhea was calculated to be 0.193 . The expected value of spatial autocorrelation for randomness was -0.0078 , with the standard deviation of 0.058 . Because the value of expected I is smaller than the observed Moran's I value, the significance test of I ( $\mathrm{z}$ test) was 3.46 . With the given value of $Z$ score, there is less than $1 \%$ likelihood that this clustered pattern could be the result of random chance. This test indicates that the prevalence of childhood diarrhea is clustered and there is strong spatial dependence across the study area. In Figure 2 (b), local indicator of spatial association (LISA) map depicts colored regions where clustering is significant. The map shows 4 types of spatial patterns based on pre-specified significances: High - High $(\mathrm{H}-\mathrm{H})$, High - Low $(\mathrm{H}-\mathrm{L})$, Low High $(\mathrm{L}-\mathrm{H})$ and Low - Low $(\mathrm{L}-\mathrm{L})$. Regions within Tete and Zambezia have HH clusters demonstrating statistically significant cluster of higher prevalence of childhood diarrhea as compared to the average of study area. However, the regions within Gaza and Cabo Delgado have L - L clusters, where these regions have statistically significant lower prevalence as compared to the average prevalence of childhood diarrhea in Mozambique.

\section{Discussion}

In this paper, data from Mozambique DHS 2011 were used to quantify the spatial dependency in the prevalence of childhood diarrhea. We began this analysis with the hypothesis that observed variation in the prevalence of childhood diarrhea is a random phenomenon. We applied 2 broad categories of detecting spatial cluster: global and local spatial autocorrelation indexes. The former measures the average tendency of data to refute spatial randomness (21) (i.e. null hypothesis), while local methods identify spatial clusters and outliers $(20,22)$. In either method, spatial autocorrelation can be considered as a tool to detect the likelihood of an event in a given region limits, or make more probable, the occurrence of an event in neighboring regions (20). Using global Moran's I index, we found spatial autocorrelation in the prevalence of diarrhea in Mozambique. Furthermore, local Moran's I index was applied to decompose the global spatial autocorrelation and to identify spatial clusters and outliers. The spatial pattern in the prevalence of childhood diarrhea in the choropleth map demonstrated a similar picture in the LISA map revealed by local Moran's I. The results of the local spatial autocorrelation analysis showed that regions in Tete and Zambezia had a higher prevalence of child- 
(a) Prevalence of Childhood Diarrhea in Mozambique

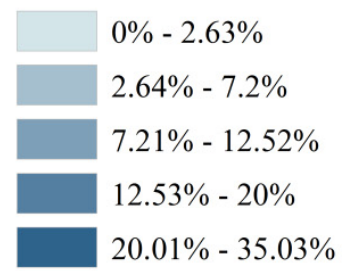

(b) LISA Cluster Map for Childhood Diarrhea in Mozambique
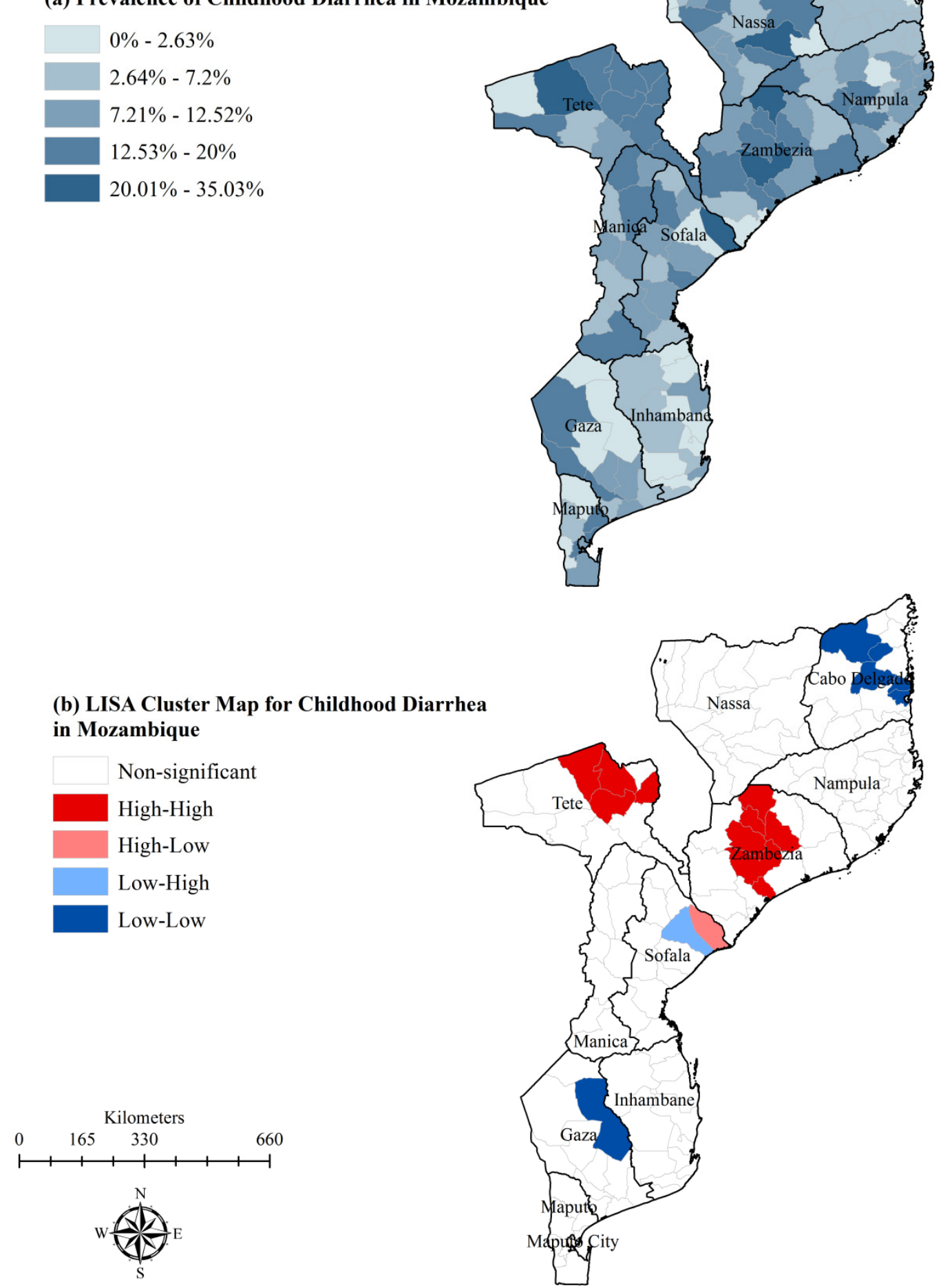

Fig. 2. (a) Choropleth map and (b) Local spatial autocorrelation (LISA) cluster map of the prevalence of childhood diarrhea in Mozambique

hood diarrhea and were locally autocorrelated based on the prevalence data. In contrast to our findings, Gujral et al found fewer cases of diarrheal diseases in lakeside regions, suggesting evidence against an endemicity of diar- rhea in these regions (23).

The observed spatial dependency was most likely resulted from spatial heterogeneity in the underlying risk factors of childhood diarrhea and its incidence in Mozam- 
bique (23). Horn's finding suggested that childhood diarrhea is more common throughout the year in coastal regions (24). The spatial clusters identified in this study are in neighboring regions of Africhol multicenter project. The regions under this project had recent cholera outbreaks (25). The fact that regions with $\mathrm{H}-\mathrm{H}$ clusters in Zambezia and outliers in Sofala state were identified near outbreak regions signifies the reintroduction of circulating pathogens in the region (26). Therefore, regions with $\mathrm{H}-\mathrm{H}$ clustering identified in LISA should be carefully studied to develop suitable strategies for controlling childhood diarrheal cases. Accordingly, for both H-H and H-L spatial clusters preventive measures, such as educating handwashing practices, adequate supply of clean drinking water, improving facilities to dispose child's stool, and introducing rotavirus vaccine, should be promoted on a broader scale to alleviate the burden of disease (27). The L-L clusters are bounded within the regions where prevalence of diarrhea is less than the average of study area. These exploratory findings are consistent with other studies from Mozambique reporting burden of childhood diarrheal diseases and its spatial distribution (24).

This study revealed regional disparity and clustering of the prevalence of childhood diarrhea across Mozambique. Moreover, these clustering patterns identified in our analysis pointed out the varying spatial distribution of prevalence of childhood diarrhea. This highlights the need of policies targeted for certain regions which are clustered together. Our results could be seen as an important firststep in understanding the complex geography of childhood diarrhea in Mozambique, and this work should be followed by in depth spatial regression analysis to better understand the causal mechanism of clustering. Our study also highlights that an updated etiologic data on diarrheal diseases and susceptibility patterns of diarrheal pathogens are required for the health ministry to design better control strategies (7). Furthermore, future studies should determine whether clusters and outliers of prevalence of childhood diarrhea are associated with specific demographic and environmental characteristics such as poverty, underprivileged household conditions, overcrowding, lack of exclusive breastfeeding, and lack of access to health care facilities (28). Finally, since the data used in this study had statistically significant spatial autocorrelation, it is warranted that any future study using prevalence data of childhood diarrhea from Mozambique from DHS 2011 should use spatial statistical methods.

Like any other research that employs spatial analyses, this study suffers from modifiable areal unit problem (MAUP). MAUP arises when data gathered and subsequently analyzed may vary from the analysis conducted at an alternative modifiable unit (29-32). Such that altered clusters and outliers' pattern would emerge if analysis would have been conducted at different areal unit.

\section{Conclusion}

We have highlighted the value of application of global and local Moran's I index for identification of clusters and outliers of childhood diarrhea in Mozambique. The method used here provides comprehensive information on the spatial distribution. Our results may help public health officials to determine appropriate allocation of health and financial resources to high-risk areas with an aim of lowering prevalence of childhood diarrhea. The use of prevalence data on the sub-district region of selected spatial clusters and outliers can be more helpful.

\section{Acknowledgments}

We express our thanks to ORC Macro International, USA, for allowing us to use datasets from MEAURE DHS archive. This study was financially supported by Tehran University of Medical Sciences.

\section{Conflict of Interests}

The authors declare that they have no competing interests.

\section{References}

1. World Health Organization. Diarrhoeal disease fact sheet. [online] 2017 [cited 2018 July]. Available from: http://www.who.int/ mediacentre/factsheets/fs330/en/.

2. Nair GB, Ramamurthy T, Bhattacharya MK, Krishnan T, Ganguly S, Saha DR, et al. Emerging trends in the etiology of enteric pathogens as evidenced from an active surveillance of hospitalized diarrhoeal patients in Kolkata, India. Gut Pathogens. 2010;2(1):4.

3. Deen JL, Von Seidlein L, Sur D, Agtini M, Lucas ME, Lopez AL, et al. The high burden of cholera in children: comparison of incidence from endemic areas in Asia and Africa. PLoS Negl Trop Dis. 2008;2(2):e173.

4. Webb C, Cabada MM. A Review on Prevention Interventions to Decrease Diarrheal Diseases' Burden in Children. Curr Trop Med Rep. 2018:1-10.

5. WHO Global Database on Child Growth and Malnutrition. Geneva: WHO.

6. World Health Organization. Report on cholera control programme in Mozambique, WHO Global Task Force on Cholera Control, 2003 Geneva WHO.

7. Mandomando IM, Macete EV, Ruiz J, Sanz S, Abacassamo F, Valles $\mathrm{X}$, et al. Etiology of diarrhea in children younger than 5 years of age admitted in a rural hospital of southern Mozambique. Am J Trop. Med Hyg. 2007;76(3):522-7.

8. IMinisterio da Saude (MISAU), Instituto Nacional de Estatística (INE), e ICF International (ICFI). Moçambique Inquérito Demográfico e de Saúde 2011.

9. Kitron U. Landscape ecology and epidemiology of vector-borne diseases: tools for spatial analysis. J Med Entomol. 1998;35(4):43545.

10. Lloyd CD. Local models for spatial analysis: CRC press; 2010.

11. Jacquez GM. Spatial cluster analysis. The handbook of geographic information science. 2008;395(416).

12. Kandala NB, Magadi MA, Madise NJ. An investigation of district spatial variations of childhood diarrhoea and fever morbidity in Malawi. Soc Sci Med 2006;62(5):1138-52.

13. Magalhaes RJS, Clements AC. Mapping the risk of anaemia in preschool-age children: the contribution of malnutrition, malaria, and helminth infections in West Africa. PLoS Med. 2011;8(6):e1000438.

14. Kazembe LN, Muula AS, Appleton CC, Kleinschmidt I. Modelling the effect of malaria endemicity on spatial variations in childhood fever, diarrhoea and pneumonia in Malawi. Int $\mathrm{J}$ Health Geogr. 2007;6(1):33.

15. Kazembe LN, Appleton CC, Kleinschmidt I. Spatial analysis of the relationship between early childhood mortality and malaria endemicity in Malawi. Geospat Health. 2007;2(1):41-50.

16. Turi KN, Christoph MJ, Grigsby-Toussaint DS. Spatial distribution of underweight, overweight and obesity among women and children: results from the 2011 Uganda Demographic and Health Survey. Int J Environ Res Public Health. 2013;10(10):4967-81.

17. Moran PA. The interpretation of statistical maps. J R Stat Soc Series B Stat Methodol. 1948;10(2):243-51.

18. Haining R, Haining RP. Spatial data analysis: theory and practice: 
Cambridge University Press; 2003.

19. Lloyd CD. Exploring population spatial concentrations in Northern Ireland by community background and other characteristics: an application of geographically weighted spatial statistics. Int J Geogr Inf Sci. 2010;24(8):1193-221.

20. Anselin L. Local indicators of spatial association-LISA. Geogr Anal. 1995;27(2):93-115.

21. Burra T, Jerrett M, Burnett RT, Anderson M. Conceptual and practical issues in the detection of local disease clusters: a study of mortality in Hamilton, Ontario. Can Geogr. 2002;46(2):160-71.

22. Kulldorff M, Tango T, Park PJ. Power comparisons for disease clustering tests. Comput Stat Data Anal. 2003;42(4):665-84.

23. Gujral L, Sema C, Rebaudet S, Taibo CLA, Manjate AA, Piarroux $\mathrm{R}$, et al. Cholera epidemiology in Mozambique using national surveillance data. J Infect Dis. 2013;208(suppl_1):S107-S14.

24. Horn LM. Precipitation Associated with Increased Diarrheal Disease in Mozambique; A Time Series Analysis 2017.

25. Baltazar CS, Langa JP, Baloi LD, Wood R, Ouedraogo I, NjanpopLafourcade BM, et al. Multi-site cholera surveillance within the African Cholera Surveillance Network shows endemicity in Mozambique, 2011-2015. PLoS Negl Trop Dis. 2017;11(10):e0005941.

26. Langa JP, Sema C, De Deus N, Colombo MM, Taviani E. Epidemic waves of cholera in the last two decades in Mozambique. J Infec Dev Ctries. 2015;9(06):635-41.

27. Nhampossa T, Mandomando I, Acacio S, Quintó L, Vubil D, Ruiz J, et al. Diarrheal disease in rural Mozambique: burden, risk factors and etiology of diarrheal disease among children aged 0-59 months seeking care at health facilities. PloS One. 2015;10(5):e0119824.

28. Das JK, Salam RA, Bhutta ZA. Global burden of childhood diarrhea and interventions. Curr Opin Infect Dis. 2014;27(5):451-8.

29. Marceau DJ, Hay GJ. Remote sensing contributions to the scale issue. Can J Remote Sens. 1999;25(4):357-66.

30. Parenteau MP, Sawada MC. The modifiable areal unit problem (MAUP) in the relationship between exposure to NO2 and respiratory health. Inter J Health Geogr. 2011;10(1):58.

31. Openshaw S. Ecological fallacies and the analysis of areal census data. Environ Plan A. 1984;16(1):17-31.

32. Schuurman N, Bell N, Dunn JR, Oliver L. Deprivation indices, population health and geography: an evaluation of the spatial effectiveness of indices at multiple scales. J Urban Health. 2007;84(4):591-603. 